\title{
Efficient identification of critical parameters affecting the small-disturbance stability of power systems with variable uncertainty
}

DOI:

10.1109/PESGM.2016.7741291

\section{Document Version}

Accepted author manuscript

Link to publication record in Manchester Research Explorer

Citation for published version (APA):

Hasan, K., Preece, R., \& Milanovic, J. V. (2016). Efficient identification of critical parameters affecting the smalldisturbance stability of power systems with variable uncertainty. In Power and Energy Society General Meeting (PESGM), 2016 https://doi.org/10.1109/PESGM.2016.7741291

\section{Published in:}

Power and Energy Society General Meeting (PESGM), 2016

\section{Citing this paper}

Please note that where the full-text provided on Manchester Research Explorer is the Author Accepted Manuscript or Proof version this may differ from the final Published version. If citing, it is advised that you check and use the publisher's definitive version.

\section{General rights}

Copyright and moral rights for the publications made accessible in the Research Explorer are retained by the authors and/or other copyright owners and it is a condition of accessing publications that users recognise and abide by the legal requirements associated with these rights.

\section{Takedown policy}

If you believe that this document breaches copyright please refer to the University of Manchester's Takedown Procedures [http://man.ac.uk/04Y6Bo] or contact uml.scholarlycommunications@manchester.ac.uk providing relevant details, so we can investigate your claim.

\section{OPEN ACCESS}




\title{
Efficient Identification of Critical Parameters Affecting the Small-Disturbance Stability of Power Systems with Variable Uncertainty
}

\author{
K. N. Hasan, R. Preece, and J. V. Milanović \\ School of Electrical and Electronic Engineering \\ The University of Manchester, Manchester, UK \\ kazi.hasan@manchester.ac.uk,robin.preece@manchester.ac.uk,milanovic@manchester.ac.uk
}

\begin{abstract}
This paper implements an efficient sensitivity analysis (SA) technique to identify and rank critically important uncertain parameters that affect the small-disturbance stability of a power system. Identification and ranking of uncertain parameters are vital in modern power system operation due to the adoption of deregulated market structure and integration of intermittent energy resources and new types of loads. Ranking of critical uncertain parameters will facilitate better operation and control with less monitoring (targeted only on the parameters of interest) by system operators and stakeholders. The Morris screening method of sensitivity analysis has been described and implemented in this paper as the most suitable for this study based on comparison with various local and global techniques which highlighted the their comparative computational complexities and simulation time requirements. All methods have been illustrated using a modified version of the 68 bus NETSNYPS test system. Illustrative results are provided considering varying levels of parameter uncertainties in order to establish not only the impact of system variability on parameter ranking, but also the robustness of the presented technique.
\end{abstract}

Index Terms-- Computational efficiency, power system analysis computing, probability distribution, sensitivity analysis, smallsignal stability, uncertainty.

\section{INTRODUCTION}

The numbers of uncertain parameters are increasing in power system operation with the adoption of intermittent renewable energy resources and new types of loads. Intermittent resources such as wind and solar power exhibit inherent randomness, whereas loads are becoming increasingly non-conventional due to increased electrification of many sectors including heating and transportation. These uncertain parameters bring challenges in system operation to maintain security and stability criteria. Probabilistic assessment tools are useful in incorporating the uncertain parameters into system stability and security assessment. As the numbers of uncertain parameters grow with the size of the power system and through increasing integration of intermittent resources, it significantly increases computational burden to consider all uncertain parameters in the evaluation of the system operation and performance. Some of these uncertain parameters could be

This work is supported by the EPSRC, UK, through the Supergen+ for HubNet project (grant number: EP/M015025/1). critical, whereas some may have little or no impact on the system operation.

Sensitivity analysis (SA) techniques can provide a framework to identify the most influential uncertain parameters. Identification of the most important uncertainties will facilitate an appropriate resource allocation guideline for system operators and stakeholders to monitor the most important parameters systematically or introduce suitable mitigation strategies where appropriate. Identification and ranking of important parameters is a vital research in power systems which has been implemented in generator ranking [1], load classification [2], voltage stability [3], PSS design [4], PMU placement [5], and frequency support [6]. Previous works [1-5] have applied 'local' linear search algorithms such as singular value decomposition, whereas a recent work [6] implemented computationally expensive 'global' methods. Screening methods - which combine to a large extent the efficiency of 'local' and accuracy of 'global' methods - are an efficient way to identify influential parameters [7-10]. Though the applications of sensitivity analysis techniques are limited in power system area, these have been extensively used in several other areas, such as flood prediction [8], urban water supply [9], hydrological modelling [10], crop model prediction [11], cancer models [12], aircraft infrared signature [13], and geolocation systems [14]. The Morris screening method has been applied in some of these previously mentioned studies to assess the importance of uncertain variables [8-10]. The derivative of system state space matrix together with left and right eigenvectors, i.e., "analytical approach", has been also used for the ranking of system parameters based on their influence on small disturbance stability [15]. The application of numerical screening techniques, however, is much more convenient (as it can be directly applied in most commercially available software packages) for a wide range of applications (not limited to small-disturbance stability) using readily available simulation outputs.

This paper presents the identification of the most influential uncertain parameters affecting the small disturbance stability of a power system through the use of an efficient screening method. The paper is organized as follows: Section II presents an overview of SA techniques and a comprehensive discussion 


\section{ACCEPTED VERSION OF THE PAPER}

of the Morris screening method. Section III discusses the test power system and probabilistic modelling of uncertain parameters. Section IV illustrates the identification and ranking of the most important uncertain parameters through the Morris screening method. It also evaluates the robustness of the ranking order by running multiple experiments with varying levels of uncertainty and different loading conditions. Research findings and conclusions are summerized in Section V.

\section{SENSITIVITY ANALYSIS TECHNIQUES}

Sensitivity analysis (SA) techniques can be used to numerically determine how input variability propagates through a computational model to its output results [9].

Three broad categories of SA techniques can be defined as: (1) local, (2) screening, and (3) global, where the computational cost and complexity increases from (1) to (3).

Local sensitivity techniques determine the local impact of an individual input parameter on the model output, by empirically calculating the partial derivatives of the output with respect to the input. In order to do so, a small variation of the input prameters is performed. One such local SA approach is the one-at-a-time (OAT) technique. This approach is easy to implement and requires low computational cost requiring just $p+1$ model evaluations for a system with $p$ uncertainties.

However, the performance of this method is insufficient when the model is nonlinear and uncertainty importance varies significantly across the range of possible uncertainty values $[7,10]$.

A screening method is used to identify the most influential parameters through its semi-global search with a lesser amount of simulation. This method qualitatively ranks the input factors according to their importance, but does not quantitatively measures the precise contribution of the parameter to the output variance. Hence, this is a trade-off between computational efficiency and accurate information. The Morris method is one such screening method. This approach is relatively easy to implement and has been shown to perform better than local SA methods $[7,8,10]$.

A global sensitivity method evaluates the importance of parameters across the full range of possible input values. Global SA methods can be subdivded further into: (1) those which are non-parametric such as correlation coefficients, (2)

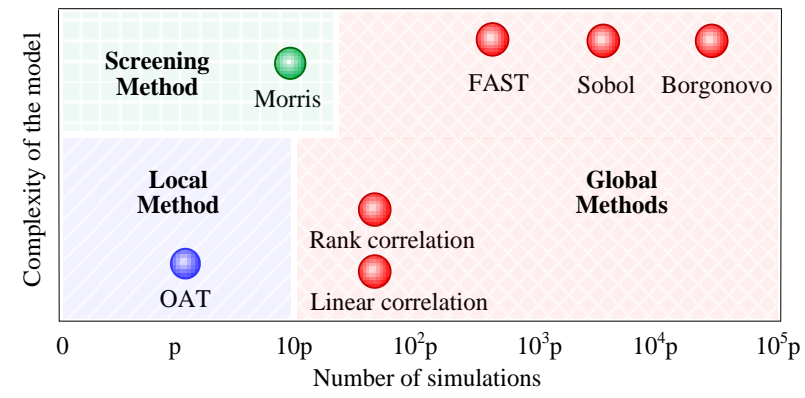

Fig. 1. Relative computational effort and complexity of the sensitivity analysis techniques. those which analyse the influence on output variance variation such as Sobol indices or the FAST (Fourier Amplitude Sensitivity Testing) method, and (3) those which analyse output probability distributions such as the Borgonovo method. These methods are complex in nature and computationally expensive $[7,10]$. The relative complexity and computational cost of different sensitivity analysis techniques are presented in Fig. 1.

When a system has a large number of input parameters and it is computationally expensive to evaluate the model, then a screening method can be the most suitable method to identify the critical parameters. Applications of the Morris method as an efficient screening method have been previously reported in the assessment of climate scenarios and hydrology $[9,10]$.

\section{A. Morris Screening Method}

The Morris method can identify and rank important variables with a limited number of model evaluations. If the variables are changed by same relative amount, the input variable which causes the largest output deviation can be considered the most important. The Morris method uses this pricinciple to identify the most significant parameters and also the degree of linear (or non-linear) influence [7, 8].

Morris method changes one variable at a time by a magnitude of $\Delta$. The standardized (or elementary) effect of a $\Delta$ change is defined as (1).

$$
E E_{p}^{i}(x)=\frac{\left[y\left(x_{1}, x_{2}, \ldots, x_{i-1}, x_{i}+\Delta, x_{i+1}, \ldots, x_{p}\right)-y(x)\right]}{\Delta}
$$

In (1), $E E_{p}^{i}$ is the elementary effect, $p$ is total number of variables, $\Delta$ is the magnitude of each step, which is a multiple of $1 /(r-1)$, and $r$ is the number of levels.

\section{1) Trajectory Construction}

The Morris method randomly changes a single variable at a time by $\Delta$, while other variables remain fixed. From this new position, the method then randomly selects another variable and makes another step of $\Delta$ in the multi-dimensional space. This process is continued until $r$ steps are completed for each variable. In this way a trajectory through the input variable search space is created, as illustrated in Fig. 2.

In the generation of the trajectory, and initial base value is randomly selected for each uncertainty ranging from $\Delta$ to $1-\Delta$. This is the base point of the creation of the trajectory.

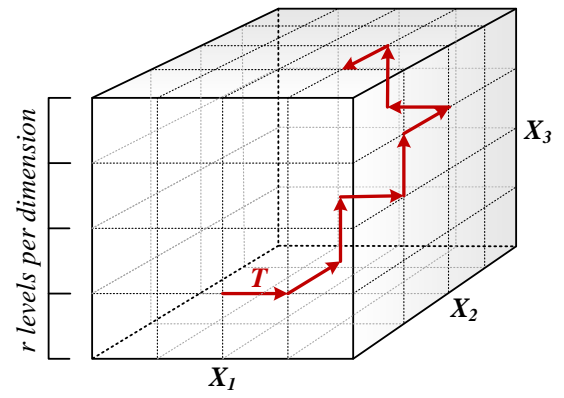

Fig. 2. Generation of Morris screening trajectory in three dimensions. 


\section{ACCEPTED VERSION OF THE PAPER}

Each step of $\pm \Delta$ is randomly selected provided the step will not exit the search space. The arrows in Fig. 2 show a possible trajectory path for the Morris method in three dimensions.

\section{2) Sensitivity Indices}

The Morris method proposes two importance measures, which are mean $\left(\mu^{*}\right)$ and standard deviation $\left(\sigma^{*}\right)$ of the elementary effects of each input variable.

$$
\begin{aligned}
& \mu^{*}=\frac{1}{r} \sum_{i=1}^{r}\left|E E_{p}^{i}\right| \\
& \sigma^{*}=\sqrt{\frac{1}{r} \sum_{i=1}^{r}\left(\left|E E_{p}^{i}\right|-\mu^{*}\right)^{2}}
\end{aligned}
$$

where, $\mu^{*}$ expresses the sensitivity strength between the $p^{\text {th }}$ input variable and the output. A high value of $\mu^{*}$ demonstrates a high contribution of the input to the output and $\sigma^{*}$ denotes the standard deviation of the distribution of the elementary effects. A variable with large $\sigma^{*}$ has a non-linear effect on the output and it has interaction with other variables $[8,9]$. A prominent characteristic of the Morris method is its low computational cost. It requires just $p \cdot r+1$ simulations, where $p$ is the number of uncertain parameters and $r$ is the number of levels per dimension (typically 4-10) [7, 8].

\section{TEST SYSTEM AND MODELLED UNCERTAINTY}

The simulation network is a substantially modified version of the NETS-NYPS test system (New England Test System New York Power System) which has 5 areas, 16 machines and 68 buses, as shown in Fig. 3. Network data, component modelling and more information of the test system are available in [16, 17].

Probabilistic system variables are wind speed, load demand and solar irradiation, which follows Weibull, normal and beta distributions, respectively, as presented in Table I with their model parameters. The normal, Weibull and beta distributions are represented through [mean $(\mu)$, standard deviation $(d)]$, [scale parameter $(\alpha)$, shape parameter $(\beta)$ ] and [shape parameter $1(a)$, shape parameter $2(b)]$, respectively. In total, 49 uncertain parameters (35 loads, 7 wind and 7 solar farms) have been modelled probabilistically. Locations of electrical loads, DFIG wind and FCC (Full Converter Connected) solar are shown in Fig. 3.

Probability density function $(p d f)$ plots of these uncertainties are presented in Fig. 4. High level of uncertainty is the base case scenario, and medium and low levels will be discussed in Section IV in order to analyze the robustness of the Morris technique. Table II presents system loading for robustness analysis which will be discussed in Section IV. Peak loading has been considered as base case scenario.

Probabilistic modelling of the input parameters and sensitivity analyses have been performed in MATLAB and OPF simulation has been solved in MATPOWER. Modal analysis has been conducted in DIgSILENT PowerFactory.

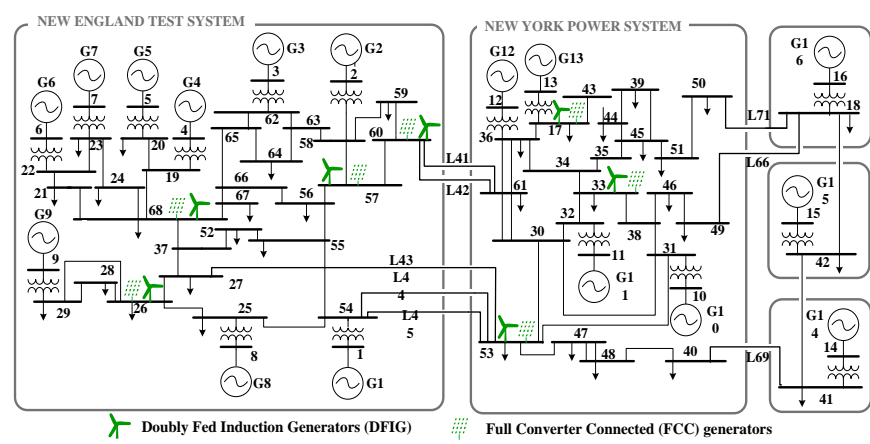

Fig. 3. Modified NETS-NYPS (New England Test System - New York Power System) network with a high amount of wind and solar generation.

TABLE I

PROBABILITY DistribUTIONS AND PROBABILISTIC MODEL PARAMETERS OF UNCERTAIN INPUT VARIABLES

\begin{tabular}{cccc}
\hline \hline $\begin{array}{c}\text { Level of } \\
\text { Uncertainty }\end{array}$ & $\begin{array}{c}\text { Load Demand } \\
\text { (Normal) }\end{array}$ & $\begin{array}{c}\text { Wind Speed } \\
\text { (Weibull) }\end{array}$ & $\begin{array}{c}\text { Solar Radiation } \\
\text { (Beta) }\end{array}$ \\
\hline High & $3 d=10 \%$ of $\mu$ & $\alpha=2.2, \beta=11.1$ & $a=13.7, b=1.3$ \\
Medium & $3 d=5 \%$ of $\mu$ & $\alpha=2.5, \beta=9.9$ & $a=17.5, b=1.3$ \\
Low & $3 d=1 \%$ of $\mu$ & $\alpha=3.1, \beta=8.8$ & $a=23.5, b=1.3$ \\
\hline \hline
\end{tabular}
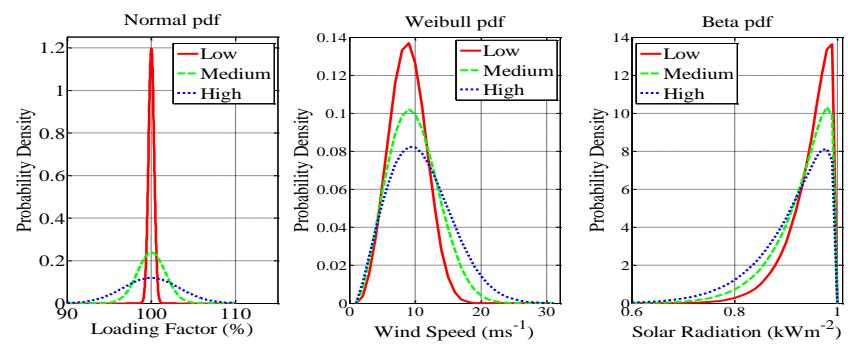

Fig. 4. Different levels of uncertainty in load, wind and solar power to replicate realistic network scenario.

TABLE II

LOADING SCENARIOS OF THE POWER SYSTEM NETWORK

\begin{tabular}{lccc}
\hline \hline \multicolumn{2}{c}{ Operational Scenarios } & System Loading & $\begin{array}{c}\text { Proportion of } \\
\text { Renewables }\end{array}$ \\
\hline Loading 1 & Peak Load & $100 \%$ & $30 \%$ \\
Loading 2 & Moderate Load & $60 \%$ & $40 \%$ \\
Loading 3 & Low Load & $30 \%$ & $60 \%$ \\
\hline \hline
\end{tabular}

\section{ILLUSTRATIVE RESULTS AND DISCUSSION}

The contour and footprints of the most critical eigenvalue in the test system is presented in Fig. 5 using results from a pure Monte Carlo probabilistic simulation. This demonstrates the variability of the critical eigenvalue with respect to the 49 uncertain system parameters. The input parameters that are most influential (and which have the highest contribution to the movement of the eigenvalues) can be identified using the Morris screening technique. 


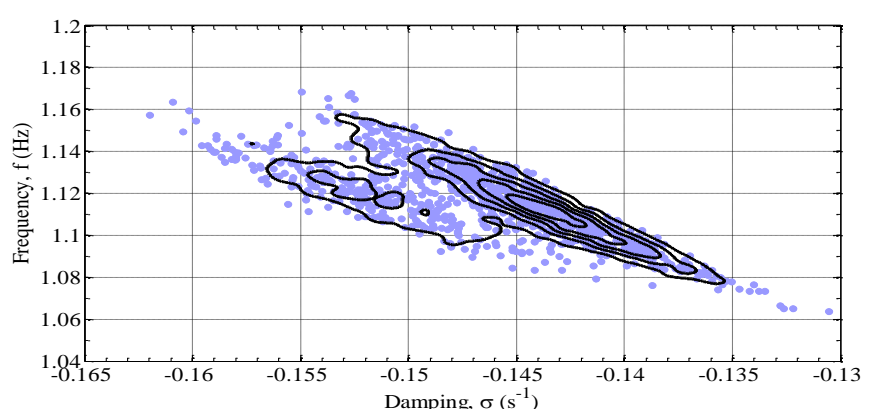

Fig. 5. Contour and footprints of a selected eigenvalue on the complex plane as affected by input parameter uncertainties.

\section{A. Sensitivity Analysis through Morris Method}

The ranking of input parameters obtained by the Morris method is shown in Fig.6 in which $\mu^{*}$ is plotted against $\sigma^{*}$. These uncertain parameter importance measures are calculated with respect to the influence on the damping (real part) of the critical eigenvalue. Based on the results shown in Fig.6, the Morris method enables the classification of the inputs in three groups: uncertain parameters having (1) negligible effects, (2) large linear effects, and (3) large non-linear effects.

Parameters with large $\mu^{*}$ scores have a high influence on the damping of the critical eigenvalue, such as uncertain input parameters 10, 11, 25 and 26 (i.e. loads $L_{17}, L_{18}, L_{41}$ and $L_{42}$ ). It can be concluded that the model output (i.e. eigenvalue damping) is largely linearly dependent on these influential inputs (particularly 10 and 11) as $\sigma^{*} \ll \mu^{*}$ for these parameters. Parameters located on the $\sigma^{*}=\mu^{*}$ line and nearby have a more non-linear influence on the output distribution. Parameters with low values of $\mu^{*}$ are considered as noninfluential.

The most influential inputs such as $L_{17}, L_{18}, L_{41}$ and $L_{42}$ require more attention and comprehensive monitoring to ensure system stability and security. The most influential 4 parameters are the 4 largest loads (6000, 2470, 1000 and 1150, respectively) presented in the NETS-NYPS power system, which is a justification of their priority ranking. The Morris method also identifies parameters which have negligible impacts on the small-disturbance stability of the system. The uncertainty associated with these parameters can be neglected during the system studies to lessen the computational burden.
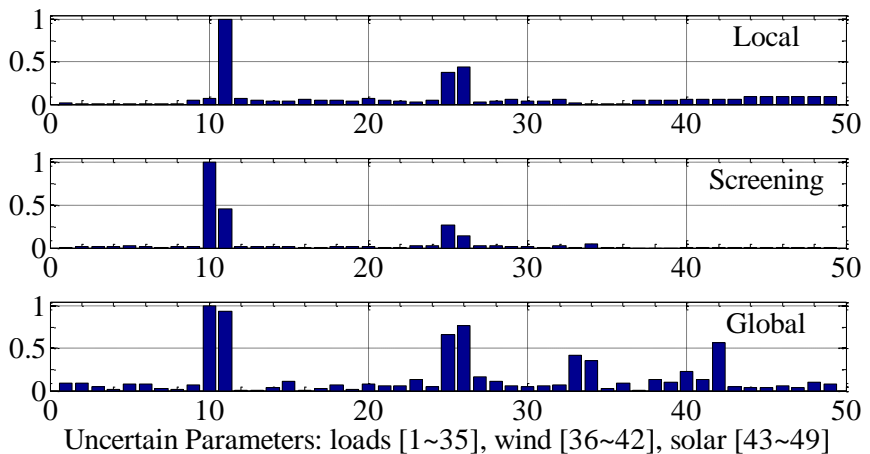

Fig. 7. Ranking of uncertain parameters through local (OAT), screening (Morris) and global (Correlation Coefficient) sensitivity analysis techniques.

\section{B. A Comparison with Local and Global Methods}

For comparison and validation, the priority ranking of the 49 uncertain parameters has also been determined using a local (OAT) and a global (Pearson correlation coefficient) ranking technique, as presented in Fig. 7. The OAT method (50 simulations, 4 mins) overvalues the importance of some parameters through its local search (compared to the global method which can be considered as a benchmark). The Correlation coefficient (1000 simulations, 40 mins) is superior to the local method but it is computationally expensive. The Morris method (246 simulations, 9 mins) can more efficiently identify the most important parameters, in particular, and shows good ranking agreement with the global method.

Wind and solar energy resources do not appear in Morris ranking, however, these come in global ranking. This is reflecting the limitation of 'semi-global' trajectory with respect to 'global' search. Further study will explore the resolution of trajectory to cover a wider space to improve the accuracy of the Morris technique. The following analyses show the impacts of levels of uncertainties and power system operating conditions on the priority ranking obtained by the Morris method.

\section{Effect of Levels of Input Parameter Uncertainties}

Different levels of input parameter uncertainties have been described in Table I with the resultant $p d f s$ of the normal, Weibull and beta distributions that have been used to represent uncertain parameters illustrated in Fig.4. Peak loading (100\%) is the base point, around which different levels of uncertainties have been modelled. The relative movements of the priority

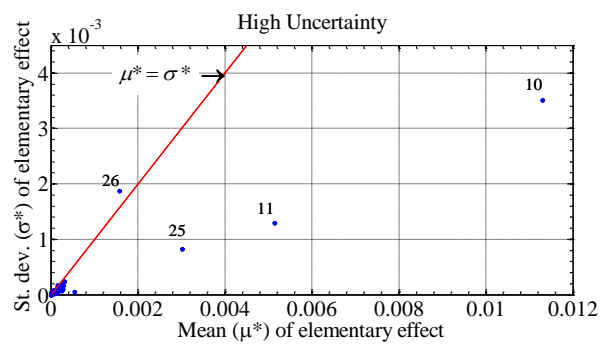

(a)

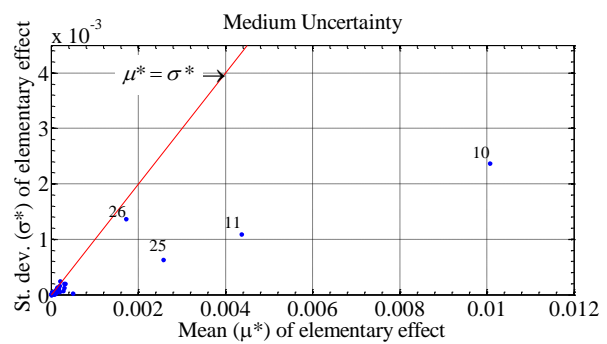

(b)

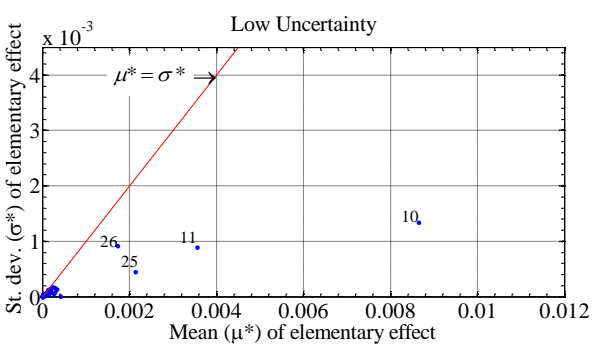

(c)

Fig. 6. Ranking of uncertain parameters obtained by Morris method, considering their relative influence on the small-disturbance stability for - (a) high uncertainty, (b) medium uncertainty, and (c) low uncertainty. 


\section{ACCEPTED VERSION OF THE PAPER}

TABLE III

PRIORITY RANKING OF TOP 5 MOST IMPORTANT PARAMETERS IDENTIFIED THROUGH MORRIS SCREENING METHOD

\begin{tabular}{|c|c|c|c|c|c|}
\hline $\begin{array}{l}\text { Ranking } \\
\text { Scenarios }\end{array}$ & 1 & 2 & 3 & 4 & 5 \\
\hline High Uncertainty & $L_{17}$ & $L_{18}$ & $L_{41}$ & $L_{42}$ & $L_{51}$ \\
\hline Med. Uncertainty & $L_{17}$ & $L_{18}$ & $L_{41}$ & $L_{42}$ & $L_{51}$ \\
\hline Low Uncertainty & $L_{17}$ & $L_{18}$ & $L_{41}$ & $L_{42}$ & $L_{51}$ \\
\hline Loading 1 & $L_{17}$ & $L_{18}$ & $L_{41}$ & $L_{42}$ & $L_{51}$ \\
\hline Loading 2 & $L_{17}$ & $L_{18}$ & $L_{41}$ & $L_{42}$ & $L_{20}$ \\
\hline Loading 3 & $L_{17}$ & $L_{18}$ & $L_{41}$ & $L_{42}$ & $L_{50}$ \\
\hline
\end{tabular}

Letters represent loads (L) presented at NETS-NYPS test system, whereas the subscripts are the bus numbers.

ranking of uncertain parameters with respect to the levels of uncertainties are presented in Table III. The priority ranking of parameters by Morris method has been minimally affected by the levels of uncertainties. Top 5 parameters always remain in the same position. Though the mean values and standard deviations of influential parameters are scaled down (as shown in Fig.6), the priority ranking always remain the same.

\section{Impact of Loading Levels}

Different power system loading conditions have been presented in Table II. System loading levels have been considered as peak $(100 \%)$, moderate $(60 \%)$ and low. While reducing the power system loading level to $60 \%$ and $30 \%$, conventional generators have been removed from service and hence the proportion of renewables has been increased to $40 \%$ and $60 \%$, respectively. The impact of the power system loading variations on the critical parameter ranking is presented in Table III. The most important 4 parameters remain the same at all system loading conditions. The $5^{\text {th }}$ parameter is changing to $L_{20}$ and $L_{50}$ for $60 \%$ and $30 \%$ loading respectively.

\section{CONCLUSIONS}

The priority ranking of the most influential input parameters which have the largest effect on power system small-disturbance stability have been presented in this paper. The parameters have been ranked using the Morris screening method. The results obtained highlight the poor performance of a local sensitivity analysis (SA) technique, whereas global methods are computationally demanding and inefficient for many large-scale applications. The Morris method offers a significant benefit in providing more accurate results than 'local' and more efficient results than 'global' SA methods. The accuracy of the Morris method lies in its trajectory exploration across multi-dimensional semi-global search space.

The robustness of the Morris-generated priority ranking has been validated in the context of uncertainty levels and system loading conditions. The robustness analyses of the priority ranking verify the reliability of the ranking method and illustrate its effectiveness across different operational scenarios. Application of the Morris method has been demonstrated using a large power system with 49 uncertain parameters. A significantly reduced computation time with respect to full global SA approaches makes it efficient and feasible for application in realistic power networks with many uncertain parameters. Advance identification of dominant uncertainties through an efficient SA technique may facilitate appropriate monitoring and control over selected parameters and ensure more secure operation of a power system. This paper provides a basis to extend the use of the Morris method as an attractive technique to apply to additional power systems planning, analysis, operation and related areas of power systems.

\section{REFERENCES}

[1] F. B. Alhasawi and J. V. Milanovic, "Ranking the Importance of Synchronous Generators for Renewable Energy Integration," IEEE Trans on Power Sys, vol. 27, pp. 416-423, 2012.

[2] A. M. Leite da Silva, J. L. Jardim, L. R. de Lima, and Z. S. Machado, "A Method for Ranking Critical Nodes in Power Networks Including Load Uncertainties," IEEE Trans on Power Sys, vol. PP, pp. 1-9, 2015.

[3] N. Amjady and M. Esmaili, "Application of a new sensitivity analysis framework for voltage contingency ranking," IEEE Trans on Power Sys, vol. 20, pp. 973-983, 2005.

[4] C. Y. Chung, K. W. Wang, C. T. Tse, and R. Niu, "Power-system stabilizer (PSS) design by probabilistic sensitivity indexes (PSIs)," IEEE Trans on Power Sys, vol. 17, pp. 688-693, 2002.

[5] M. Dehghani, B. Shayanfard, and A. R. Khayatian, "PMU Ranking Based on Singular Value Decomposition of Dynamic Stability Matrix," IEEE Trans on Power Sys, vol. 28, pp. 2263-2270, 2013.

[6] R. Preece and J. V. Milanovic, "Assessing the Applicability of Uncertainty Importance Measures for Power System Studies," IEEE Transactions on Power Systems, vol. PP, pp. 1-9, 2015.

[7] A. Saltelli, K. Chan, and E. M. Scott, Sensitivity Analysis: John Wiley \& Sons, 2008.

[8] Bertrand Iooss and P. Lematre, A review on global sensitivity analysis methods: Springer, 2015.

[9] D. M. King and B. J. C. Perera, "Morris method of sensitivity analysis applied to assess the importance of input variables on urban water supply yield - A case study," Journal of Hydrology, vol. 477, pp. 17-32, 2013.

[10] X. Song, J. Zhang, C. Zhan, Y. Xuan, M. Ye, and C. Xu, "Global sensitivity analysis in hydrological modeling: Review of concepts, methods, theoretical framework, and applications," Journal of Hydrology, vol. 523, pp. 739-757, 2015.

[11] H. Varella, M. Guérif, and S. Buis, "Global sensitivity analysis measures the quality of parameter estimation: The case of soil parameters and a crop model," Environmental Modelling \& Software, vol. 25, pp. 310319,2010

[12] W. Zhihui, T. S. Deisboeck, and V. Cristini, "Development of a sampling-based global sensitivity analysis workflow for multiscale computational cancer models," IET Systems Biology, vol. 8, pp. 191197, 2014

[13] S. Lefebvre, A. Roblin, S. Varet, and G. Durand, "A methodological approach for statistical evaluation of aircraft infrared signature," Reliability Engineering \& System Safety, vol. 95, pp. 484-493, 2010.

[14] E. Ward, "Global sensitivity analysis of terrain effects in geolocation systems," IEEE Trans on Aero \& Elec Sys, vol. 51, pp. 2039-46, 2015.

[15] J. V. Milanovic, I. A. Hiskens, and V. A. Maslennikov, "Ranking loads in power systems-comparison of different approaches," IEEE Trans on Pow Sys, vol. 14, pp. 614-619, 1999.

[16] G. Rogers, Power System Oscillations: Norwell: Kluwer Academic Publishers, 2000.

[17] B. Pal and B. Chaudhuri, Robust Control in Power Systems. New York: Springer Inc., 2005. 